\title{
ORBITS AND CHARACTERS ASSOCIATED TO HIGHEST WEIGHT REPRESENTATIONS
}

\author{
DAVID H. COLLINGWOOD
}

(Communicated by Jonathan M. Rosenberg)

\begin{abstract}
We relate two different orbit decompositions of the flag variety. This allows us to pass from the closed formulas of Boe, Enright, and Shelton for the formal character of an irreducible highest weight representation to closed formulas for the distributional character written as a sum of characters of generalized principal series representations. Otherwise put, we give a dictionary between certain Lusztig-Vogan polynomials arising in Harish-Chandra module theory and the Kazhdan-Lusztig polynomials associated to a relative category $\mathscr{O}$ of Hermitian symmetric type.
\end{abstract}

\section{INTRODUCTION}

Thirty-five years ago, Harish-Chandra showed how each complex structure on a Hermitian symmetric space $G / K$ gives rise to a family of irreducible highest weight representations of $G$. Several people have studied these representations, typically from one of two viewpoints, depending on the representation theoretic category in which one wishes to work. On the one hand, these representations lie in a category $\mathscr{O}_{P}$, containing generalized Verma modules induced from a maximal parabolic subalgebra determined by the implicit complex structure. On the other hand, these highest weight modules are admissible $(g, K)$-modules and as such lie in the category $\mathscr{H} \mathscr{C}$ of Harish-Chandra modules for $G$. Each category contains a family of standard modules with unique irreducible quotients and one can formulate a character problem. In category $\mathscr{O}_{P}$, we consider formal characters and the problem is to write the formal character of an irreducible highest weight module as a sum of characters of generalized Verma modules with certain coefficients; in this case, one constructs a Hecke module $\mathscr{M}_{P}$, which gives rise to a collection of recursively defined Kazhdan-Lusztig polynomials in one variable (say $u$ ) and the desired coefficients are (up to sign) given by these polynomials evaluated at $u=1$. In the category $\mathscr{H} \mathscr{C}$, we consider the distribution character of a globalization of each irreducible highest weight module, and the problem is write this character as a sum of characters of generalized principal series representations (i.e. induced from discrete

Received by the editors September 4, 1990 and, in revised form, October 15, 1990.

1980 Mathematics Subject Classification (1985 Revision). Primary 22E46, 22 E47.

Supported in part by a National Security Agency grant MDA904-90-H-4041 and The University of Washington Graduate School Research Fund. 
series representations of $G$ ) with certain coefficients; here, a Hecke module $\mathscr{M}_{K}$ associated to the pair $(G, K)$ gives rise to a collection of recursively defined Lusztig-Vogan polynomials and the desired coefficients are (up to sign) given by these polynomials evaluated at $u=1$.

The point of this note is to show that every Lusztig-Vogan polynomial associated to an irreducible highest weight representation coincides with one of the Kazhdan-Lusztig polynomials associated to a Hermitian symmetric category $\mathscr{O}_{P}$. Works of Boe, Enright-Shelton, and Lascoux-Schultzenberger provide us with closed formulas for these Kazhdan-Lusztig polynomials (see [2, 6]), hence we obtain closed formulas for the Lusztig-Vogan polynomials associated to an irreducible highest weight module. From a general viewpoint, this coincidence is striking, since the complexity of the Hecke module structure on $\mathscr{M}_{K}$ makes that of $\mathscr{M}_{P}$ pale by comparison. However, if we adopt the geometric viewpoint toward representation theory, we can think of representations as corresponding to orbits in the flag variety (modulo some issues regarding flat connections on the orbits). The representation theory in the category $\mathscr{O}_{P}$ (resp. $\mathscr{H} \mathscr{C}$ ) is related to the P-orbit (resp. K-orbit) structure on the flag variety $\mathscr{B}$ of all Borel subalgebras of the complexified Lie algebra of $G$, where $\mathbf{P}$ (resp. $\mathbf{K}$ ) is a parabolic subgroup of $\mathbf{G}$ corresponding to the complex structure on $G / K$ (resp. is the complexification of a maximal compact subgroup $K$ of $G$; we assume throughout that $G$ is a matrix group so that such a $K$ exists). The parabolic subgroup $\mathbf{P}$ has Levi decomposition $\mathbf{P}=\mathbf{K N}$ and hence every $\mathbf{P}$-orbit is $\mathbf{K}$-stable and itself a union of $\mathbf{K}$-orbits. Using known dictionaries between character formulas and the Euler characteristics of certain intersection cohomology complexes (defined over characteristic $p$ ), we can see that the desired relationship between Kazhdan-Lusztig polynomials and Lusztig-Vogan polynomials amounts to a good description of the connection between $\mathbf{K}$-orbits and $\mathbf{P}$-orbits. The thrust of this note is to give just such a connection. Our analysis depends on three ingredients: (i) a recent paper of Richardson-Springer [12], which gives a combinatorial description of the Bruhat order on symmetric varieties; (ii) the essential geometric lemma in [4] used to compute the Hecke module structure on $\mathscr{M}_{P}$ and Vogan's analogous calculation for $\mathscr{M}_{K}$ in [14]; and (iii) a very special property of the Bruhat posets $W^{P}$ attached to a Hermitian symmetric pair. The main results and proofs are given in the next section. Section 2 illustrates our results in the case of $\mathrm{SL}_{2} \mathbb{R}$ and $\mathrm{SU}(2,2)$. In a separate joint paper with B. Boe [3], we show how these ideas allow one to carry over the "orthogonal sets" of Enright-Shelton to the Harish-Chandra module setting.

\section{Main Results}

Let $(G, K)$ be an irreducible Hermitian symmetric pair, with $G$ assumed to be linear. Then $K$ is a maximal compact subgroup of $G$; the corresponding complexifications are denoted by boldface letters $\mathbf{K}$ and $\mathbf{G}$. If we fix a complex structure on the symmetric space $G / K$, then this determines a parabolic subgroup $\mathbf{P}$ of $\mathbf{G}$ with the property that the compact dual symmetric space $G^{\mathbf{v}} / K=\mathbf{G} / \mathbf{P}$; see [7]. Denote the Lie algebra of $\mathbf{P}$ by $\rho$ and the complexified Lie algebras of $G$ and $K$ by $g$ and $k$, respectively. Then we have a Levi decomposition $\rho=\not k \oplus n$. Let $\mathscr{B}$ denote the flag variety $\mathbf{G} / \mathbf{B}$, where $\mathbf{B}$ is a Borel subgroup of $\mathbf{G}$ compatible with $\mathbf{P}$. Then $\mathbf{P}$ and $\mathbf{K}$ both act on $\mathscr{B}$, 
decomposing it into a finite disjoint union of orbits; see [1,9]. Let $W^{P}$ denote the minimal length right coset representatives of $W_{P} \backslash \mathrm{W}$, where $W\left(\right.$ resp., $W_{P}$ ) is the Weyl group associated to $g$ (resp. $k$ ). As is well known, we have a P-orbit decomposition

$$
\mathscr{B}=\bigcup_{w \in \mathrm{W}^{P}} \mathscr{O}_{w} .
$$

Next, let $\mathrm{V}$ index the $\mathbf{K}$-orbits in $\mathscr{B}$ and write the $\mathbf{K}$-orbit decomposition

$$
\mathscr{B}=\bigcup_{\delta \in \mathrm{V}} \mathscr{V}_{\delta} .
$$

Since $\mathbf{K}$ is a subgroup of $\mathbf{P}$, each $\mathbf{P}$-orbit $\mathscr{O}_{w}$ is $\mathbf{K}$-stable and itself is a union of $\mathbf{K}$-orbits. Since $\mathbf{K}$ is connected, each $\mathbf{P}$-orbit $\mathscr{O}_{w}$ contains a unique open dense $\mathbf{K}$-orbit $\mathscr{V}_{\delta_{w}}$, for some $\delta_{w} \in \mathrm{V}$. If $X$ is a subset of $\mathscr{B}$, we denote by $\bar{X}$ the Zariski closure of $X$ in $\mathscr{B}$. Recall that $W^{P}$ is a partially ordered set under the Bruhat order $\leq: y \leq w$ if and only if $\overline{\mathscr{O}}_{w} \supseteq \mathscr{O}_{y}$. On the set $\mathrm{V}$ we introduce the closure ordering $\leq_{K}: \overline{\mathscr{V}}_{\delta} \supseteq \mathscr{V}_{\gamma}$ if and only if $\gamma \leq_{K} \delta$. The order relations $\leq$ and $\leq_{K}$ make $W^{P}$ and $\mathrm{V}$ into partially ordered sets, which possess length functions $l$ : define $l(\cdots)$ on $\mathrm{V}$ (resp. $\mathrm{W}^{P}$ ) by $l\left(\mathscr{V}_{\delta}\right)=\operatorname{dim}_{\mathbb{C}} \mathscr{V}_{\delta}-t$, (resp. $l(w)=\operatorname{dim}_{\mathbb{C}} \mathscr{O}_{w}-r$ ), where $t=$ the dimension of the closed K-orbits (resp. $r$ is the dimension of the closed P-orbit) in $\mathscr{B}$. In our setting, $t=r=$ $\operatorname{dim}_{\mathbb{C}} \mathscr{B}-(1 / 2)\left(\operatorname{dim}_{\mathbb{R}} G / K\right)$.

(1.1) Lemma (Closure lemma). If $w \in W^{P}$, then $\overline{\mathscr{V}_{\delta_{w}}}=\bigcup_{y \leq w} \mathscr{O}_{w}=\overline{\mathscr{O}_{w}}$.

Remarks. (i) Our proof of (1.1) has the advantage of simultaneously producing an algorithm for computing the decomposition of a P-orbit into K-orbits, using the coherent continuation calculus; see remarks following Lemma (1.2).

(ii) This lemma is asserting that the closures of certain $\mathbf{K}$-orbits are unions of P-orbits; this is not true in general. The simplest example occurs in $\mathrm{SL}_{2} \mathbb{R}$. If we fix a holomorphic structure on $G / K$ corresponding to $\mathbf{P}$, then we see that one of the closed $\mathbf{K}$-orbits $\mathscr{V}$ coincides with the closed P-orbit, while the other closed K-orbit $\mathscr{V}^{\sharp}$ is not a union of P-orbits; we can choose the opposite holomorphic structure corresponding to the opposite parabolic subgroup $\overline{\mathbf{P}}$ and show $\mathscr{V}^{\sharp}$ is a closed $\overline{\mathbf{P}}$-orbit. But in general, varying the complex structure is not enough. For example, consider the case of $\operatorname{SU}(2,2)$ in $(2.2)$; the K-orbit labeled $\mathscr{V}_{\delta_{15}}$ is not a union of $\mathbf{P}$-orbits for any $\mathbf{P}$.

Using (1.1) we describe the decomposition of any $\mathscr{O}_{w}$ as a union of $\mathbf{K}$-orbits. To do so, for each $w \in W^{P}$, we introduce a subset $S_{w}$ of $S$, where $S$ is the Coxeter basis of $W$ (relative to our fixed $\mathbf{B}$ ): $S_{w}=\{s \in S \mid w s<w\}$; a subset of the tau invariant of $w$.

(1.2) Lemma (Decomposition lemma). Let $w \in W^{P}$, then we have $\mathscr{O}_{w}=$ $\bigcup_{\gamma \in \mathrm{V}_{w}} \mathscr{V}_{\gamma}$, where $\mathrm{V}_{w}=\left\{\gamma \in \mathrm{V} \mid \gamma \leq_{K} \delta_{w}\right.$ and $\gamma \mathbb{Z}_{K} \delta_{w s}$ for all $\left.s \in \mathrm{S}_{w}\right\}$.

Remarks. This result gives a closed formula for the $\mathbf{K}$-orbit decomposition of a P-orbit, in terms of the $\leq_{K}$ order. On the other hand, as noted above, the proof of (1.1) gives an algorithmic answer that does not depend on knowledge of the full $\leq_{K}$ order, rather, only knowledge of its underlying weak order; this is often more useful in explicit calculations.

We define a map $\mathbb{A}: \mathrm{V} \rightarrow \mathrm{W}^{P}$ by the rule that $\mathbb{A}(\delta)=z$, where $\mathscr{O}_{z}$ is the unique P-orbit containing $\mathscr{V}_{\delta}$. Lemma (1.2) gives a means for computing the 
map $\mathbb{A}$, in terms of the order relation $\leq_{K}$. A combinatorial description of the order relation $\leq_{K}$ is given in [12]. Combinatorial models for $V$ have been floating around in the folklore for some time. In the classical cases, see [10]. In the exceptional cases EIII and EVII (using the labeling in [7, p. 534]), one can use the theory in [15]; see [3] for more on these. In $\S 3$ we describe the map $\mathbb{A}$ in the cases of $\mathrm{SL}_{2} \mathbb{R}$ and $\mathrm{SU}(2,2)$.

This leads us to our final consequence regarding the distribution character of an irreducible highest weight module. Although not essential, to simplify the statement and prevent obscuring the main point, we assume $G$ is one of the groups $\mathrm{SU}(p, q), \mathrm{SO}_{e}(2,2 n), \mathrm{SO}^{*}(2 n)$, EIII, or EVIII; for the other Hermitian symmetric cases $\mathrm{Sp}_{n} \mathbb{R}$ and $\mathrm{SO}_{e}(2,2 n+1)$, there is a slight difference

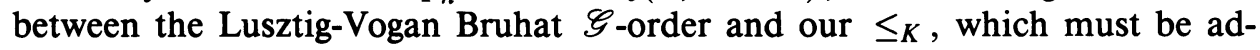
dressed. Now [4, (3.5)-(3.12)] describes how to construct a Hecke module $\mathscr{M}_{P}$, which depends on the $\mathbf{P}$-orbit structure and certain $\mathbb{P}^{1}$-calculations. In a similar spirit (and prior to [4]), Vogan constructed a Hecke module $\mathscr{M}_{K}$ associated to the block of the trivial representation (see [13, §9.2]), which depends on the $\mathbf{K}$-orbit structure and analogous $\mathbb{P}^{1}$-calculations; see $[14, \S 5 ; 8]$. Each of the Hecke modules $\mathscr{M}_{P}$ and $\mathscr{M}_{K}$ gives rise to a collection of recursively defined polynomials in one variable with integer coefficients, denoted

$$
\left\{P_{y, w}(u) \in \mathbb{Z}[u]: y, w \in \mathrm{W}^{P}\right\} \quad \text { and } \quad\left\{Q_{\gamma, \delta}(u) \in \mathbb{Z}[u]: \delta, \gamma \in \mathrm{V}\right\}
$$

we refer to these as Kazhdan-Lusztig polynomials and Lusztig-Vogan polynomials, respectively. We are interested in relating these two sets of polynomials. Recall that $Q_{\gamma, \delta_{w}}(u) \neq 0$ only if $\gamma \leq_{K} \delta_{w}$.

\section{(1.3) Lemma (Comparison lemma). If $\gamma \leq_{K} \delta_{w}$, then $Q_{\gamma, \delta_{w}}(u)=P_{\mathrm{A}(\gamma), w}(u)$.}

Recall that $\mathrm{V}$ is in one-to-one correspondence with the block of the trivial representation of $G$ and hence so too with a subset of the set $\mathscr{D}$ of $[8,14]$. As described in [14], we may associate to each $\delta \in \mathscr{D}$ a generalized principal series representation $\pi(\delta)$ having a unique irreducible quotient $\bar{\pi}(\delta)$; i.e. $\delta$ determines a triple $\left(P_{\delta}=M_{\delta} A_{\delta} N_{\delta}, \sigma_{\delta}, \nu_{\delta}\right)$ consisting of a Langlands decomposition of a cuspidal parabolic subgroup $P_{\delta}$ of $G$, a relative discrete series representation $\sigma_{\delta}$ of $M_{\delta}$ and a "negative" character $\nu_{\delta}$ on $A_{\delta}$. We use the notation $\boldsymbol{\theta}_{G}(\cdots)$ to denote the distribution character of a Harish-Chandra module. We now have the following representation theoretic consequence of (1.3):

(1.4) Corollary (Character formula). For each $w \in \mathrm{W}^{P}$, we have

$$
\boldsymbol{\Theta}_{G}\left(\bar{\pi}\left(\delta_{w}\right)\right)=\sum_{\gamma \leq K}(-1)^{l\left(\delta_{w}\right)+l(\gamma)} P_{\mathbf{A}(\gamma), w}(1) \boldsymbol{\Theta}_{G}(\pi(\gamma)) .
$$

As noted above, this gives closed formulas for these character expressions. Bearing in mind the different index sets, this should be compared with the classical character formula

$$
\boldsymbol{\Theta}\left(\bar{\pi}\left(\delta_{w}\right)\right)=\sum_{y \leq w}(-1)^{l(w)+l(y)} P_{y, w}(1) \boldsymbol{\Theta}\left(V_{y}\right)
$$

coming from category $\mathscr{O}_{P}$, where $V_{y}$ are generalized Verma modules (relative to $\rho)$. We illustrate this in $(2.2)$ in the setting of $\mathrm{SU}(2,2)$.

Finally, it is of interest to know when (if ever) all of the nonzero LusztigVogan polynomials associated to $\bar{\pi}\left(\delta_{w}\right)$ are the constant polynomial 1. Recall the notion of a Kostant module introduced in [5]. 
(1.5) Corollary (Rationality result). Fix an irreducible Harish-Chandra module $\bar{\pi}\left(\delta_{w}\right), w \in \mathrm{W}^{P}$. The following are equivalent:

(i) $\bar{\pi}\left(\delta_{w}\right)$ is a Kostant module;

(ii) for every $\gamma \in \mathrm{V}, \gamma \leq_{K} \delta_{w}, Q_{\gamma, \delta_{w}}(u)=1$.

We now describe the proofs of (1.1)-(1.3). To begin, suppose $s \in \mathrm{S}$ and consider the $\mathbb{P}^{1}$-fibration $\pi_{s}: \mathscr{B} \rightarrow \mathscr{P}_{s}$ to the variety of parabolics of type $s$. If $X$ is a subset of $\mathscr{B}$, we define $\hat{X}=\pi_{s}^{-1}\left(\pi_{s}(X)\right)$; this "hat" operation depends on $s$, which is always clear from context. Combining [4, $(3.12), 14,(5.1)]$ we see that $\widehat{\mathscr{O}}_{w}$ is a union of one or two P-orbits and $\widehat{\mathscr{V}}_{\delta}$ is a union of one, two, or three K-orbits, for all $w \in \mathrm{W}^{P}$ and $\delta \in \mathrm{V}$.

We prove by induction on the length of $\delta_{w}$ the following statement:

$$
\overline{\mathscr{V} \delta_{w}}=\bigcup_{y \leq w} \mathscr{O}_{y} \text {. }
$$

If $l\left(\delta_{w}\right)$ is minimal, the $\mathscr{V}_{\delta_{w}}$ is a closed dense $\mathbf{K}$-orbit inside the closed P-orbit $\mathscr{O}_{w}$ and $l\left(\delta_{w}\right)=l(w)$; hence they coincide and $(*)$ holds. Assume $\mathscr{V}_{\delta_{w}}$ is not a closed K-orbit. Then there exists $s \in \mathrm{S}$ such that $w s<w$ and $\delta_{w s}<_{K} \delta_{w}$. By the "one-step-property" of Richardson-Springer [12, (7.7)],

$$
\overline{\mathscr{V}_{\delta_{w}}}=\bigcup_{\gamma \leq K} \widehat{\mathscr{Q}}_{\delta_{w s}} .
$$

But $\bigcup_{\gamma \leq_{k} \delta_{w s}} \hat{\mathscr{O}}_{\gamma}=\left(\bigcup_{\gamma \leq_{k} \delta_{w s}} \mathscr{O}_{\gamma}\right)^{\wedge}=\left(\overline{\mathscr{V}_{\delta_{w s}}}\right)^{\wedge}=\left(\bigcup_{y \leq w s} \mathscr{O}_{y}\right)^{\wedge}=\bigcup_{y \leq w s} \hat{\mathscr{O}}_{y}$, by induction. A careful look at $\$ 5$ of [12] shows us that the "one-step-property" holds for P-orbits and we have $\bigcup_{y \leq w s} \widehat{\mathscr{O}}_{y}=\overline{\mathscr{O}}_{w}=\bigcup_{y \leq w} \mathscr{O}_{y}$; this is $(*)$ and $(1.1)$ is proved.

We assert that for each $z \in \mathrm{W}^{P}$,

$$
\mathscr{V}_{\gamma} \subseteq \overline{\mathscr{O}_{z}} \text { if and only if } \gamma \leq_{K} \delta_{z} .
$$

If $\gamma \leq_{K} \delta_{z}$, then by definition and (1.1), $V_{\gamma} \subseteq \overline{\mathscr{V}_{\delta_{z}}}=\overline{\mathscr{O}_{z}}$. On the other hand, if $\mathscr{V}_{\gamma} \subseteq \overline{\mathscr{O}_{z}}=\bigcup_{y \leq z} \mathscr{O}_{y}$, then $\mathscr{V}_{\gamma} \subseteq \mathscr{O}_{y}$, some $y \leq z$. We previously noted that $\delta_{y}$ is the unique open dense orbit inside $\mathscr{O}_{y}$, so $\gamma \leq_{K} \delta_{y}$. We claim that $\delta_{y} \leq_{K} \delta_{z}$, which would establish $(* *)$. This follows from the fact that the simple reflections that generate the weak order on $\mathrm{W}^{P}$ likewise gives weak relations in the $\leq_{K}$ order between the $\delta_{u}$ 's, $u \in \mathrm{W}^{P}$. In other words, suppose we have a chain

$$
u<u s_{i(1)}<u s_{i(1)} s_{i(2)}<\cdots<u s_{i(1)} \cdots s_{i(k)}
$$

in $\mathrm{W}^{P}$, with $s_{i(j)} \in \mathrm{S}$. Then we have

$$
\delta_{u}<_{K} \delta_{u s_{i(1)}}<_{K} \delta_{u s_{i(1)} s_{i(2)}}<K \cdots<_{K} \delta_{u s_{i(1)} \cdots s_{i(k)}} .
$$

Next by (1.1), we see that

$$
\overline{\mathscr{V}_{\delta_{w}}}=\bigcup_{y \leq w} \mathscr{O}_{y}=\mathscr{O}_{w} \cup\left(\bigcup_{y<w ; l(y)=l(w)-1} \mathscr{O}_{y}\right) \cup\left(\bigcup_{y<w ; l(y)<l(w)-1} \mathscr{O}_{y}\right) .
$$

It is a special property of the posets $\mathrm{W}^{P}$ that $\{y \mid y<w$ and $l(y)=l(w)-1\}=$ $\left\{w s \mid s \in \mathrm{S}_{w}\right\}$, where $\mathrm{S}_{w}$ is the set defined prior to (1.2). This amounts to the 
following special fact for Hermitian symmetric Bruhat posets: the weak order determined by $S$ on $W^{P}$ and the standard order it generates on $W^{P}$ coincide; see $[6,11]$. So, we obtain

$$
\overline{\mathscr{V}_{\delta_{w}}}=\mathscr{O}_{w} \cup\left(\bigcup_{s \in \mathrm{S}_{w}} \overline{\mathscr{O}_{w s}}\right) .
$$

If we now recall $(* *)$ and let $\gamma \leq_{K} \delta_{w}$, it follows that $\mathscr{V}_{\gamma} \subseteq \mathscr{O}_{w}$ if and only if $\mathscr{V}_{\gamma} \nsubseteq \overline{\mathscr{O}_{w s}}$ for all $s \in \mathrm{S}_{w}$ if and only if $\gamma \mathbb{Z}_{K} \delta_{w s}$ for all $s \in \mathrm{S}_{w}$; this proves (1.2).

Fix a prime $p \neq 2$ and a prime power $q=p^{r}$, for which $\mathbf{G}, \mathbf{P}, \mathbf{B}$ and all the above orbits have "good reduction" to varieties $\mathbf{G}_{q}, \mathbf{P}_{q}, \mathbf{B}_{q}$, etc., all defined over $\mathbf{k}_{q}=$ an algebraic closure of the finite field $\mathbb{F}_{q}$ of $q$ elements; such is true for almost all prime powers $q$. As in [4] we obtain

$$
\left[I C\left(\overline{\mathscr{O}_{w}}\right)\right]=\sum_{y \leq w} P_{y, w}(u) \mathbf{1}_{\mathscr{O}_{y}},
$$

where $\mathbf{1}_{\mathscr{O}_{y}}$ is a one-dimensional $\mathbf{P}_{q}$-equivariant sheaf of one-dimensional $l$-adic vector spaces on $\mathscr{O}_{y}$ and $\left[I C\left(\overline{\mathscr{O}_{w}}\right)\right]$ is the Euler characteristic of the intersection cohomology complex attached to the orbit closure; see the discussion preceeding $[4,(3.28)]$. It is important to note that there are no "negative terms" in this characteristic, because of the parity result on intersection cohomology. This takes place in the setting $\operatorname{Perv}(\mathbf{P})$ of perverse $l$-adic sheaves constructible with respect to a $\mathbf{P}$-orbit stratification. If we work instead with a category $\operatorname{Perv}(\mathbf{K})$ of perverse $l$-adic sheaves constructible with respect to the $\mathbf{K}$-orbit stratification on $\mathscr{B}$, as in [8], then

$$
\left[I C\left(\overline{\mathscr{V}_{\delta_{w}}}\right)\right]=\sum_{\gamma \leq_{K} \delta_{w}} Q_{\gamma, \delta_{w}}(u) \mathbf{1}_{\mathscr{V}_{\gamma}} .
$$

By $(1.1), \overline{\mathscr{V}_{\delta_{w}}}=\bigcup_{y \leq w} \mathscr{O}_{w}=\overline{\mathscr{O}_{w}}$ and $I C\left(\overline{\mathscr{O}_{w}}\right)$ lie in the category $\operatorname{Perv}(\mathbf{K})$. We can then use (1.2) to compare terms and arrive at (1.3).

Remark. T. A. Springer pointed out the following alternate argument for (1.3): First, from generalities about perverse sheaves, the perverse extension $I C\left(\mathbf{1}_{\mathscr{O}_{w}}\right)$ of the constant sheaf $\mathbf{1}_{\mathscr{O}_{w}}$ on $\mathscr{O}_{w}$ (i.e. the intersection cohomology complex of $\left.\overline{\mathscr{O}}_{w}\right)$ is the same as the perverse extension of the constant sheaf on the open dense subset $\mathscr{V}_{\delta_{w}}$. One now notes that the restriction of $I C\left(\mathbf{1}_{\mathscr{O}_{w}}\right)$ to all $\mathscr{O}_{y}$ with $y \leq w$ is constant, so the restriction of $I C\left(\mathbf{1}_{\mathscr{O}_{w}}\right)$ to $\mathscr{V}_{\gamma}\left(\gamma \leq_{K} \delta_{w}\right)$ is constant, and is the same as the restriction of $I C\left(\mathbf{1}_{\mathscr{O}_{w}}\right)$ to $\mathscr{O}_{\mathbf{A}(\gamma)}$; this implies $Q_{\gamma, \delta_{w}}(u)=P_{\mathrm{A}(\gamma), w}(u)$.

\section{EXAMPLES}

(2.1) The case of $\mathrm{SL}_{2}(\mathbb{R})$. Throughout this paragraph, assume $G=\mathrm{SL}_{2} \mathbb{R}$. In Table 1 we have parametrized the $\mathbf{P}$ and $\mathbf{K}$ orbit decompositions of $\mathscr{B}$. We have labeled the weak order relations in the posets $W^{P}$ and $V$ with the letter " $s$ ", referring to the unique simple reflection in $S$. The levels of the poset are sympathetic to the underlying length function $l$ on each poset. Using the 
TABLE 1. P-orbits and K-orbits for $\mathrm{SL}_{2} \mathbb{R}$.

\begin{tabular}{|c|c|c|c|c|}
\hline$W^{P}$ & & $V$ & & length \\
\hline $\mathscr{O}_{s}$ & & $\mathscr{V}_{\delta_{1}}$ & & 1 \\
\hline$s \mid$ & & & & \\
\hline $\mathscr{O}_{e}$ & $\mathscr{V}_{\delta_{+}}$ & & $\mathscr{V}_{\delta_{-}}$ & 0 \\
\hline
\end{tabular}

notation of Table 1, if $\bar{\pi}\left(\delta_{+}\right)$is a holomorphic discrete series for the complex structure determined by $\mathbf{P}$, the map $\mathbb{A}$ is determined by: $\mathscr{O}_{e}=\mathscr{V}_{\delta_{+}} ; \mathscr{O}_{s}=$ $\mathscr{V}_{\delta_{-}} \cup \mathscr{V}_{\delta_{1}}$.

(2.2) The case of $\mathrm{SU}(2,2)$. Throughout this paragraph, assume $G=\mathrm{SU}(2,2)$. In Table 2 we have parametrized the $\mathbf{P}$ and $\mathbf{K}$ orbit decompositions of $\mathscr{B}$. We have labeled the weak order relations in the posets $W^{P}$ and $V$ with solid lines and an accompanying index $i$, referring to the standard enumeration of the simple reflections $s_{i}$ in $\mathrm{S}$ given by the labeled Dynkin diagram

$$
\underset{s_{1}}{0-s_{2}}-s_{3}^{-0}
$$

As noted in one of the above proofs, the weak order on $\mathrm{W}^{P}$ coincides with the standard order. In the case of $\leq_{K}$, we have drawn in the additional order relations with dotted lines. The levels of the poset are sympathetic to the underlying length function $l$ on each poset. Using the notation of Table 2 , if $\bar{\pi}\left(\delta_{4}\right)$ is a holomorphic discrete series for the complex structure determined by $\mathbf{P}$, we see the map $\mathbb{A}$ is determined by Table 3 .

Finally, we want to translate (1.3) in this setting. Consider the K-orbit $\mathscr{V}_{\delta_{20}}$ and the P-orbit $\mathscr{O}_{s_{2} s_{1} s_{3}}$ in Table 2. From [4, (3.24)] or [6] we find

$$
P_{y, s_{2} s_{1} s_{3}}(u)= \begin{cases}1 & \text { if } y \in\left\{s_{2} s_{1} s_{3}, s_{2} s_{1}, s_{2} s_{3}, s_{2}\right\} \\ 1+u & \text { if } y=e, \\ 0 & \text { otherwise. }\end{cases}
$$

From (1.3) and Table 3 it follows that

$$
Q_{\gamma, \delta_{20}}(u)= \begin{cases}1 & \text { if } \gamma=\delta_{j}, \\ & j \in\{2,3,5,6,8,9,10,11,12,15,16,17,20\} \\ 1+u & \text { if } \gamma=\delta_{4} \\ 0 & \text { otherwise }\end{cases}
$$


TABle 2. P-orbits and K-orbits for $\mathrm{SU}(2,2)$.

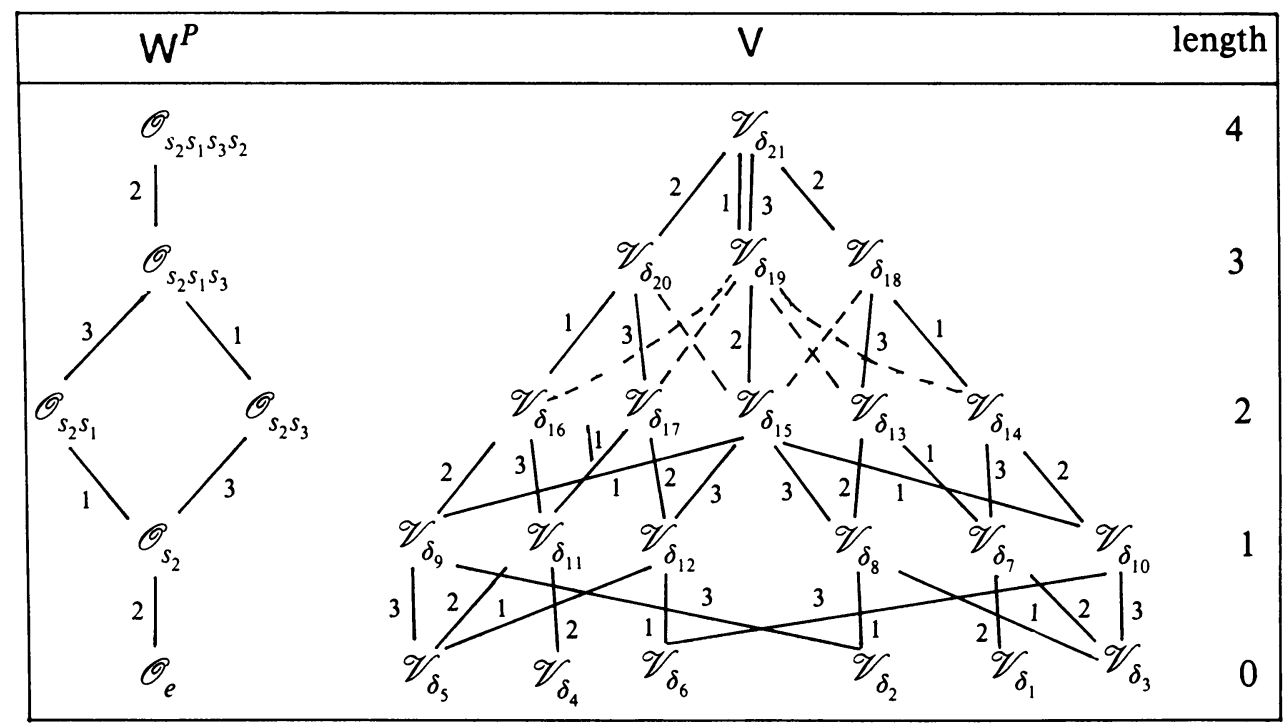

TABle 3. Decomposition of P-orbits into K-orbits.

\begin{tabular}{|ll|}
\hline P-orbit & K-orbit decomposition \\
\hline $\mathscr{O}_{s_{2} s_{1} s_{3} s_{2}}$ & $\mathscr{V}_{\delta_{21}} \cup \mathscr{V}_{\delta_{19}} \cup \mathscr{V}_{\delta_{18}} \cup \mathscr{V}_{\delta_{13}} \cup \mathscr{V}_{\delta_{14}} \cup \mathscr{V}_{\delta_{7}} \cup \mathscr{V}_{\delta_{1}}$ \\
$\mathscr{O}_{s_{2} s_{1} s_{3}}$ & $\mathscr{V}_{\delta_{20}} \cup \mathscr{V}_{\delta_{15}} \cup \mathscr{V}_{\delta_{8}} \cup \mathscr{V}_{\delta_{10}} \cup \mathscr{V}_{\delta_{3}}$ \\
$\mathscr{O}_{s_{2} s_{1}}$ & $\mathscr{V}_{\delta_{17}} \cup \mathscr{V}_{\delta_{12}} \cup \mathscr{V}_{\delta_{6}}$ \\
$\mathscr{O}_{s_{2} s_{3}}$ & $\mathscr{V}_{\delta_{16}} \cup \mathscr{V}_{\delta_{9}} \cup \mathscr{V}_{\delta_{2}}$ \\
$\mathscr{O}_{s_{2}}$ & $\mathscr{V}_{\delta_{11}} \cup \mathscr{V}_{\delta_{5}}$ \\
$\mathscr{O}_{e}$ & $\mathscr{V}_{\delta_{4}}$ \\
\hline
\end{tabular}

\section{ACKNOWLEDGMENT}

The author would like to thank T. Springer, R. Richardson, B. Boe, and the referee for their careful comments.

\section{REFERENCES}

1. I. Bernstein, I. Gel'fand and S. Gel'fand, Schubert cells and the cohomology of the spaces $G / P$, Russian Math. Surveys, no. 28, (1973), 1-26.

2. B. Boe, Kazhdan-Lusztig polynomials for Hermitian symmetric spaces, Trans. Amer. Math. Soc. 309 (1988), 279-294.

3. B. Boe and D. Collingwood, Enright-Shelton theory and Vogan's problem for generalized principal series, preprint. 
4. L. Casian and D. Collingwood, The Kazhdan-Lusztig conjecture for generalized Verma modules, Math. Z. 195 (1987), 581-600.

5. D. Collingwood, The n-homology of Harish-Chandra modules: generalizing a theorem of Kostant, Math. Ann. 272 (1985), 161-187.

6. T. Enright and B. Shelton, Categories of highest weight modules: applications to classical Hermitian symmetric pairs, Mem. Amer. Math. Soc., no. 367, Amer. Math. Soc., Providence, R.I., 1987.

7. S. Helgason, Differential geometry, Lie groups and symmetric spaces, Pure Appl. Math. 80 (1978).

8. G. Lusztig and D. Vogan, Singularities of closures of $K$-orbits on flag manifold, Invent. Math. 71 (1983), 365-370.

9. T. Matsuki, The orbits of affine symmetric spaces under the action of minimal parabolic subgroups, J. Math. Soc. Japan 31 (1979), 331-357.

10. T. Matsuki and T. Oshima, Embeddings of discrete series into principal series, preprint.

11. R. Proctor, Classical Bruhat orders and lexicographic shellability, J. Algebra 77 (1982), 104-126.

12. R. Richardson and T. Springer, The Bruhat order on symmetric varieties, Geom. Dedicata 35 (1990), 389-436.

13. D. Vogan, Representations of reductive Lie groups, Birkhäuser, Boston, 1980.

14. __ Irreducible characters of semisimple Lie groups III: proof of the Kazhdan-Lusztig conjecture in the integral case, Invent. Math. 71 (1983), 381-417.

15. __ Irreducible characters of semisimple Lie groups IV: character multiplicity duality, Duke Math. J. 49 (1982), 943-1073.

Department of Mathematics, University of Washington, Seattle, Washington 98195 